\title{
FTO expression is associated with the occurrence of gastric cancer and prognosis
}

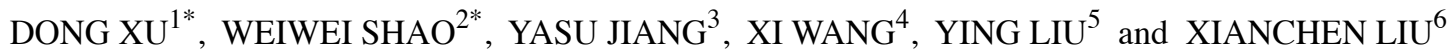 \\ ${ }^{1}$ Department of General Surgery, Affiliated Hospital of Nantong University, Nantong, Jiangsu; \\ ${ }^{2}$ Department of General Surgery, The Fourth Affiliated Hospital of Nantong University, Nantong University, Yancheng, \\ Jiangsu; ${ }^{3}$ Department of General Surgery, The Second Affiliated Hospital of Nantong University, Nantong University; \\ ${ }^{4}$ Medical College of Nantong University; ${ }^{5}$ Department of General Surgery, Nantong Geriatric Rehabilitation Hospital; \\ ${ }^{6}$ Department of Radiation Oncology, Affiliated Hospital of Nantong University, Nantong, Jiangsu, P.R. China
}

Received February 28, 2017; Accepted July 28, 2017

DOI: 10.3892/or.2017.5904

\begin{abstract}
Fat mass and obesity associated (FTO) is a proteincoding gene. FTO gene is an obesity related gene, also known as the obesity gene. It has been reported previously that FTO is associated with a variety of malignant cancers, such as breast, thyroid and endometrial cancer. The aim of the present study was investigate the FTO expression of human gastric cancer and to investigate its clinical value. FTO expression was determined by immunohistochemical analysis with tissue microarrays in GC tissues and corresponding adjacent nontumor tissues. Moreover, the results in protein and mRNA level were confirmed by the real-time PCR and western blot analysis. The relationship between the FTO expression and the pathological characteristics of GC patients was also explored. In addition, by using MTT, clone formation and Transwell assays, we studied the effects of FTO expression on biological function of GC cells in vitro. The Kaplan-Meier method and the log-rank test were used to compare the overall survival rate between the FTO high-expression group and the low-expression group. We affirmed repeatedly upregulation of FTO expression in both protein and mRNA levels in GC tissues compared to corresponding adjacent non-tumor tissues. Immunohistochemistry by tissue microarray of FTO expression was remarkably increased in GC tissues (72 of $128,56.3 \%$ ) compared with adjacent non-tumor tissues (24 of $62,38.7 \%)$. FTO expression level was closely related to low differentiation $(\mathrm{P}<0.001)$, lymph node metastasis $(\mathrm{P}=0.029)$.
\end{abstract}

Correspondence to: Dr Xianchen Liu, Department of Radiation Oncology, Affiliated Hospital of Nantong University, No. 20 Xisi Road, Nantong, Jiangsu 226001, P.R. China

E-mail: xianchenliunt@sina.com

${ }^{*}$ Contributed equally

Abbreviations: FTO, fat mass and obesity associated; GC, gastric carcinoma; TNM, tumor node metastasis

Key words: fat mass and obesity associated gene, gastric cancer, prognosis
The expression of FTO was positively correlated with TNM stage $(\mathrm{P}<0.001)$. The Kaplan-Meier analysis showed that high FTO expression was significantly associated with poor prognosis in GC patients. Downregulation of FTO expression significantly inhibited the proliferation, migration and invasion of GC cell lines. On the contrary, overexpression of FTO promoted the proliferation, migration and invasion of GC cell lines. This study indicates that FTO expression may have an important role in promoting the occurrence of GC, and it may be an vital molecular marker in the diagnosis and prognosis of GC patients.

\section{Introduction}

Gastric cancer (GC) is the fifth most common cancer and the third most common cause of cancer-related death worldwide $(1,2)$. Although the incidence of GC has been declining for several decades, GC is one of the most common cancers in China, accompanied by a high incidence and mortality, approximately accounting for $10 \%$ of all malignancies (3). The treatment strategies for gastric cancer have made great progress, however, the prognosis of gastric cancer is still poor; due to most cases being diagnosed in an advanced stage, the 5-year survival rate is only $20-30 \%$ (4). Currently known major risk factors for GC include Helicobacter pylori infection, living environment, diet, genetic and immune factors and chronic stomach diseases (5). There have been advancements in the molecular biomarkers utilised in the cancer detection, furthermore, in the development of therapeutic agents based on the target genes for a few types of solid tumors including GC (6). With the development of biotechnology, the molecular mechanisms and alterations that lead to initiation and progression of GC, including multiple genetic and molecular alterations and mutations have been revealed (7). These biomarkers can help us make early diagnosis and predict the prognosis of GC patients.

FTO also known as ALKBH9, it is localized on chromosome 16q12.2, FTO belongs to the non-heme Fe II/ $\alpha$ KG-dependent dioxygenase AlkB family proteins that also includes ABH1 to ABH8 (8). After identification of a fusedtoe mutant mouse whose phenotype results from a $1.6-\mathrm{Mb}$ 
deletion of six genes, including FTO, the FTO gene was first cloned (9). In 2007, FTO was described as the first gene in association with the common obesity. FTO is an AlkB-like 2-oxoglutarate-dependent nucleic acid demethylase with a strong preference for 3-methylthymidine and 3-methyluracil single-stranded DNA and RNA (10). It has been reported that FTO can oxidize demethylate $\mathrm{m}-3 \mathrm{~T}$ and $\mathrm{m}-3 \mathrm{U}$ in singlestranded DNA (ssDNA) and single-stranded RNA (ssRNA) in vitro (11). The expression of FTO mRNA is extensive in different human tissues indicates that it may be involved in important biological processes $(11,12)$. Although obesity is a basic biological mechanism of the risk of the development of cancer it is not fully understood, recent studies have shown that the FTO gene may associate with cancer risk (13), such as breast (14), thyroid (15) and endometrial cancer (16).

To date, no research has been reported on whether FTO is associated with GC. The aim of this study was to investigate the FTO expression in GC patient specimens and to appraise the clinicopathological implications of FTO expression in GC. Our efforts are aimed at discovering the potential influence of FTO in carcinogenesis and progression of GC.

\section{Materials and methods}

Tissue specimen. Gastric cancer (GC) specimens were collected from 128 patients with primary gastric cancer during surgery in Hospital Affiliated of Nantong University from January 2010 to February 2011, and the patients were enrolled in this study. After surgical resection, randomly selected carcinoma adjacent tissue specimens and GC tissues were obtained from 128 patients. The carcinoma adjacent tissues were assessed microscopically for the presence of normal cells and absence of dysplastic cells, and taken $>3 \mathrm{~cm}$ distance from the tumor margin. Immediately the fresh sample was divided into two parts, frozen in liquid nitrogen, one was maintained at $-80^{\circ} \mathrm{C}$ until use real-time PCR and western blot analysis, the other was embedded in paraffin after fixation in $10 \%$ formalin (24-48 h) fixed for immunohistochemical diagnosis. Clinical data were also collected on sex, age, tumor size, TNM stage, and lymph node metastasis. Distant metastasis was determined by radiological examination. Staging and grading referred to the classification of International Union Against Cancer Criteria (UICC). The patients who participated in the study did not receive radiotherapy or chemotherapy before surgery. This study was conducted with the approval of the institutional ethics board of the Hospital Affiliated of Nantong University.

Western blot analysis. Paired cancer tissue and carcinoma adjacent tissue of 24 cases were randomly chosen from 128 gastric cancer patients for western blot analysis. The whole protein was extracted by lysis buffer which contained protease inhibitors (Promega, Madison, WI, USA). The 10\% sulfate polyacrylamide gel electrophoresis (SDS-PAGE) was used to isolate equal amounts of protein, and these proteins were then transferred into a polyvinylidene fluoride (PVDF) membrane. Then the membranes were blocked for $2 \mathrm{~h}$ with $5 \%$ non-fat milk in TBST (Tris-buffered saline containing 0.1\% Tween-20) and incubated with the primary antibodies overnight at $4^{\circ} \mathrm{C}$; monoclonal mouse anti-human $\mathrm{FTO}$ at 1:1,000 dilution (Abcam, Cambridge, UK) or monoclonal mouse anti- $\beta$-actin as internal reference, at 1:2,000 dilution (SigmaAldrich, St. Louis, MO, USA). The membranes were washed three times in TBST, for $5 \mathrm{~min}$ each time. Then, incubated with IRDye $680 \mathrm{CS}$-conjugated goat anti-mouse secondary antibody (1:1,000 dilution; Sigma-Aldrich) for $2 \mathrm{~h}$ at room temperature according to the manufacturer's instructions. Eventually signals were scanned with an Odyssey Infrared Imaging system (LI-COR Biosciences, Lincoln, NE, USA) and analyzed with PDQuest 7.2.0 software (Bio-Rad Laboratories, Hercules, CA, USA).

Immunohistochemical staining and evaluation. A total of 128 GC tissues, 62 matched adjacent non-cancerous tissues specimens were prepared and used in this study. We used tissue microarray (TMA) system (Quick-Ray UT06; Unitma Co., Ltd., Seoul, Korea) in the Department of Clinical Pathology. Core tissue biopsies ( $2 \mathrm{~mm}$ in diameter) were taken from individual paraffin-embedded sections and arranged in recipient paraffin blocks. TMA specimens were cut into $4 \mu \mathrm{m}$ sections and placed on super frost-charged glass microscope slides. TMA analysis was used as a quality control for hematoxylin and eosin staining. Tissue sections were deparaffinized and rehydrated in graded ethanol. Antigen retrieval was performed by boiling sections in ethylene diaminetetra-acetic acid buffer, $\mathrm{pH}$ 6.0, for $3 \mathrm{~min}$ in a pressure cooker. Endogenous peroxidase activity was quenched with $3 \%$ hydrogen peroxide for $30 \mathrm{~min}$. TMA slides were stained using monoclonal mouse anti-FTO antibody (Abcam) at $4^{\circ} \mathrm{C}$ overnight. Then, by incubation with a horseradish-peroxidase-conjugated goat anti-mouse secondary antibody (Sigma-Aldrich) at $37^{\circ} \mathrm{C}$ for $30 \mathrm{~min}$. Slides were then processed using horseradish peroxidase and 3,3-diaminobenzidine chromogen solution and counterstained with hematoxylin. The semiquantitative $\mathrm{H}$-score method was used to convert the expression of FTO to continuous intensity values, based on both the staining intensity and the percentage of cells at that intensity. According to the traditional semiquantitative pathology scoring, staining intensity was scored as follows: 0 (-, no staining), 1 (+, weak staining), 2 (++, moderate staining), or 3 (+++, intense staining). The percentage of cells stained at a certain intensity was determined and multiplied by the intensity score to generate an intensity percentage score. The final staining score of each tissue sample was the sum of the four intensity percentage scores, and these scores ranged from 0 (no staining) to 300 (100\% of cells with +++ staining intensity).

Real-time PCR. Firstly, the TRIZol (Gibco, Grand Island, NY, USA) was used to extract total RNAs from tumor tissue samples. Then quantitative real-time PCR was performed using HotStart-IT SYBR-Green qPCR Master Mix (2X; USB Corp., Cleveland, OH, USA). In the light of the HotStart-IT protocol, $25 \mu 1$ actions were run of with $2 \mu \mathrm{l}$ cDNA. We perform RT-PCR experiments in a LightCycler 480 system (Roche Applied Sciences). PCR steps: first hot start at $95^{\circ} \mathrm{C}$ for $10 \mathrm{~min}$; then 40 cycles of amplification/quantification at $95^{\circ} \mathrm{C}$ for $10 \mathrm{sec}$, next $60^{\circ} \mathrm{C}$ for $30 \mathrm{sec}$ and $72^{\circ} \mathrm{C}$ for $30 \mathrm{sec}$ during the time fluorescence was measured. Melting curve analysis was implemented using continuous fluorescence acquisition from 65 to $97^{\circ} \mathrm{C}$. These cycling parameters produced single amplicons for both primer sets used on the basis of the presence of a 
single melt peak. We use the $\beta$-actin as the internal reference. The primer sequences are as follows: a 169-bp segment of the FTO gene 5'-TGGTGTCCCAAGAAATCGTG-3' (sense) and 5'-TGCAGGCCGTGAACCAC-3' (antisense), a 107-bp segment of the $\beta$-actin gene 5'-AACTTCCGTTGCTGCCAT-3' (sense) and 5'-TTTCTTCCACAGGGCTTTG-3' (antisense). All quantitative real-time PCRs were repeated 3 times for each gene, and each sample was done in triplicate.

Cell culture. Four human GC cell lines (BGC823, MKN45, SGC7901 and AGS) and the human normal stomach epithelial mucosa cell line (GES) from the Cell Bank of the Committee on Type Culture Collection of the Chinese Academy of Sciences (Shanghai, China). These cell lines were grown in Dulbecco's modified Eagle's medium (DMEM; Invitrogen, Carlsbad, CA, USA) or RPMI-1640 medium (Gibco) containing with $10 \%$ fetal bovine serum (FBS; HyClone Laboratories, Inc., Logan, UT, USA) and were cultured in humidified incubator at $37^{\circ} \mathrm{C}$ with $5 \% \mathrm{CO}_{2}$.

Construction of plasmid and transfection. The siRNA oligos for FTO were designed and purchased from Shanghai GenePharma Co., Ltd., (Shanghai, China). The targeting sequence was FTO-siRNA1: GCAGTGTATCTGAGGAGCTCCATAA, FTO-siRNA2: CGGTATCTCGCATCCTCATTGGTAA and FTO-siRNA3: TCAGCGGTGGCAGTGTACAGTTATA. A FTO overexpression plasmid (pcDNA3.1-FTO) containing the coding sequence was constructed using PCR-generated fragments and pcDNA 3.1(+) vector. Then the PCR products were cloned into the mammalian expression vector pcDNA 3.1(+). All constructs were confirmed by DNA sequence analysis. We use the stable transfectant of the pcDNA 3.1(+) vector as the control group. During the course of transfection, the FTO siRNA and pcDNA 3.1(+)-FTO expression plasmids were transfected into GC cells using Lipofectamine 2000 (Invitrogen) according to the manufacturer's instructions. The western blot assay was used to evaluate the level of FTO expression after transfection.

Cell viability assay and Transwell assay. MTT assay was used to assess the cell viability. Firstly, we use the 96-well plates at $5 \times 10^{3}$ cells/well in complete medium for the cells. The cells were cultured for $24 \mathrm{~h}$. Next, the culture medium was replaced with a medium which contained $10 \%$ FBS. Then, $10 \mu \mathrm{l}$ MTT was added to each corresponding test and the cells were kept in culture for $4 \mathrm{~h}$. Finally, all samples were measured at $490 \mathrm{~nm}$ spectrophotometric absorbance.

Cell migratory capacity was confirmed by Transwell assay (BD Biosciences, San Jose, CA, USA) according to the manufacturer's instructions. These transfected cells were harvested $24 \mathrm{~h}$ after transfection. Then $3.0 \times 10^{5}$ transfected cells or untreated cells were added to each upper insert in serum-free medium. To the matched lower chamber, $500 \mu \mathrm{l}$ of medium containing $10 \%$ FBS was added. After incubation, non-migrated cells were removed from the upper surface of the Transwell membrane with a cotton swab, and the migrated cells on the lower membrane surface were fixed in methanol. Lastly, $0.1 \%$ crystal violet was used to stain cells, then photographed and counted. The above experiments were performed in triplicate and repeated three times.
Table I. FTO expression compared in gastric cancer and adjacent non-tumor tissues.

\begin{tabular}{|c|c|c|c|c|}
\hline \multirow[b]{2}{*}{ Clinical parameters } & \multirow[b]{2}{*}{$\mathrm{N}$} & \multicolumn{2}{|c|}{$\begin{array}{c}\text { FTO } \\
\text { expression }\end{array}$} & \multirow[b]{2}{*}{ P-value } \\
\hline & & $\begin{array}{l}\text { Low } \\
\text { level }\end{array}$ & $\begin{array}{l}\text { High } \\
\text { level }\end{array}$ & \\
\hline Gastric cancer tissues & 128 & 56 & 72 & $0.023^{\mathrm{a}}$ \\
\hline Adjacent non-tumor tissues & 62 & 38 & 24 & \\
\hline
\end{tabular}

${ }^{\mathrm{a}} \mathrm{P}<0.05$ was considered statistically significant. FTO, fat mass and obesity associated.

Colony formation assay. Cells $\left(5 \times 10^{4} /\right.$ well) were plated in a 24-well plate after transfection. After $24 \mathrm{~h}$, the cells collected and seeded $(1,000-1,500 /$ well) in a fresh 6-well plate for 12 days. Surviving colonies (>50 cells/colony) were counted after fixed with methanol/acetone (1:1) and stained with 5\% gentian violet (ICM Pharma, Singapore). After washing three times with phosphate-buffered saline (PBS) to remove excess dye, the cells were photographed and counted. The experiment was carried out in triplicate wells three times.

Statistical analysis. All data were analyzed using statistical analyses performed using the SPSS 17.0 (SPSS, Inc., Chicago, IL, USA). The relationship between the FTO expression level and the clinicopathological characteristics was subjected to $\chi^{2}$ analysis. Survival analysis was calculated using the KaplanMeier method and curves were assessed using the log-rank test. Cox's proportional hazards model was used to carry out the multivariate analysis of several prognostic factors. The results are presented as the mean \pm SD of at least three independent experiments and $\mathrm{P}<0.05$ was considered to be statistically significant.

\section{Results}

FTO expression in primary gastric tumors. The expression of FTO was determined by TMA-based immunohistochemistry (IHC) studies. We showed that the expression level of FTO in GC tissues and adjacent tissues were different, FTO expression was significantly elevated in GC patients but negative or low in non-tumor tissues (Fig. 1). Positive staining was predominantly localized in the nucleus of GC cells. Overall, FTO expression was positive (FTO ++ or FTO +++) in 72 of $128 \mathrm{GC}$ tissues $(56.3 \%)$, the carcinoma adjacent tissues (38 of $62,61.3 \%$ ) were mostly negative of FTO expression (FTOor FTO+). Overall, GC tissues had high FTO expression as compare with the adjacent non-cancerous tissues $(\mathrm{P}=0.023$; Table I).

The above results were further tested by western blot analysis in 24 random pairs of GC and corresponding carcinoma adjacent tissues. Furthermore, the representative western blot results in 6 cases are shown in Fig. 2A. The results show that FTO protein level was upregulated in the GC samples $(75 \%$, 18 of 24) compared with corresponding carcinoma adjacent tissues. The average FTO protein level in 24 gastric carcinoma 


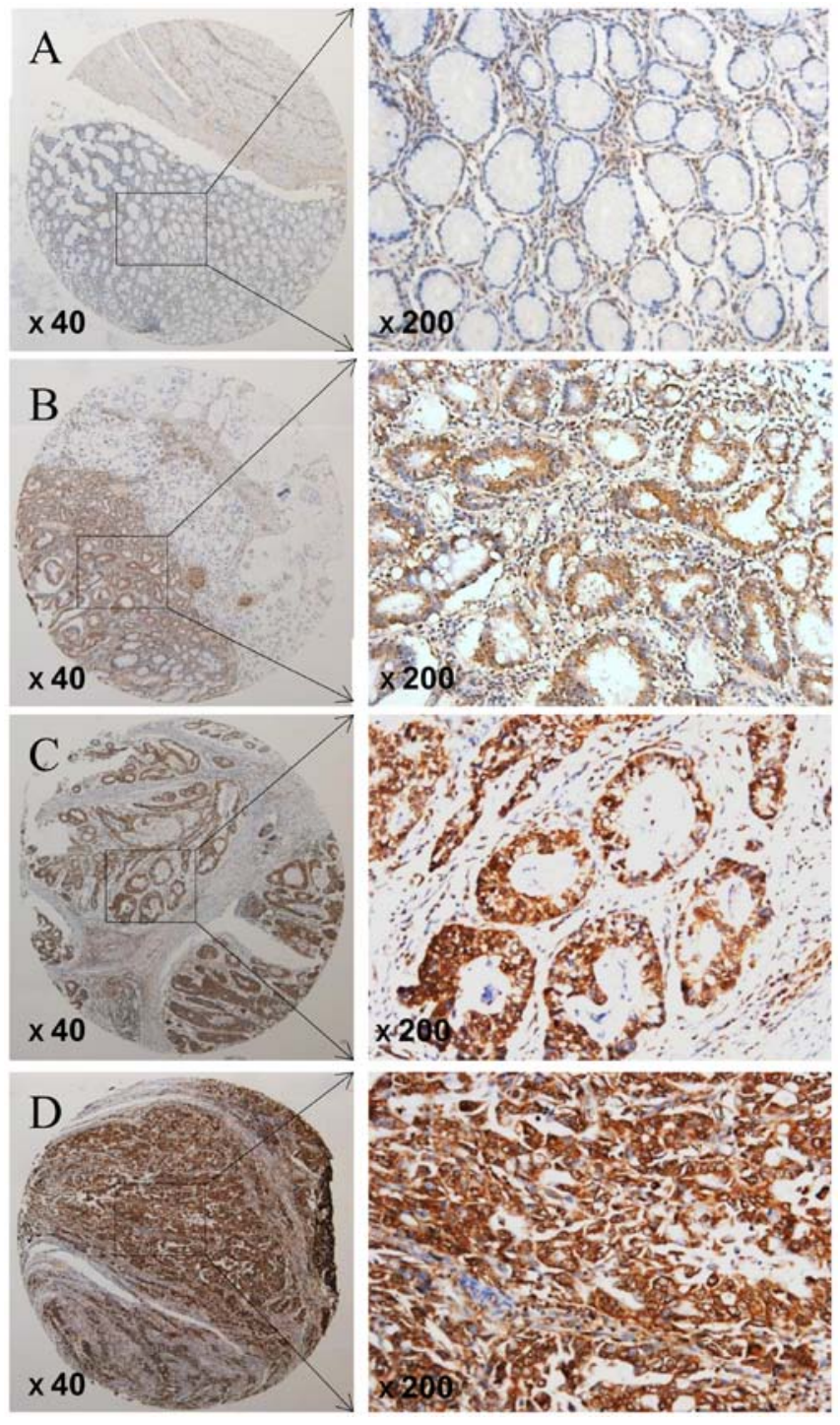

Figure 1. Representative patterns of FTO expression in GC tissues and adjacent non-cancerous tissue as determined with IHC analysis with tissue microarrays. (A) Negative staining in adjacent non-tumor tissues, scored as FTO (-). (B) Weak staining in well-differentiated GC tissues, scored as FTO (+). (C) Moderate staining in moderately differentiated GC tissues, scored as FTO (++). (D) Strong staining in poorly differentiated GC tissues, scored as FTO (+++). Original magnification, $\mathrm{x} 40$ or $\mathrm{x} 200$.

tissues was significantly higher than that in corresponding carcinoma adjacent tissues ( $\mathrm{P}=0.024$; Fig. 2B). Real-time PCR also was used to examine the mRNA expression of FTO in 36 primary GC tissues and their corresponding adjacent normal tissues selected randomly. As shown in Fig. 2C, the average expression of FTO mRNA level was significantly higher in GC tissues compared with adjacent non-cancerous tissues $(\mathrm{P}<0.001)$.

FTO expression is correlated to clinicopathological factors in GC patient survival. We use the $\chi^{2}$ test to analyze the correlation between the FTO expression in GC tissues and various clinicopathological characteristics of GC patients are listed in Table II. High expression of FTO was significantly correlated with poor differentiation $(\mathrm{P}<0.001)$, lymph node metastasis $(\mathrm{P}=0.029)$ and high TNM stage $(\mathrm{P}<0.001)$. But the FTO expression was not significantly correlated with sex, age,
Table II. Clinicopathological correlation of FTO expression in gastric cancer patients.

\begin{tabular}{|c|c|c|c|c|}
\hline \multirow[b]{2}{*}{ Clinical parameters } & \multirow[b]{2}{*}{$\begin{array}{c}\text { Total } \\
(n=128)\end{array}$} & \multicolumn{2}{|c|}{ FTO expression } & \multirow[b]{2}{*}{$\mathrm{P}$-value } \\
\hline & & $\begin{array}{c}\text { Low } \\
\text { level } \\
(n=56)\end{array}$ & $\begin{array}{c}\text { High } \\
\text { level } \\
(n=72)\end{array}$ & \\
\hline Sex & & & & 0.929 \\
\hline Male & 68 & 30 & 38 & \\
\hline Female & 60 & 26 & 34 & \\
\hline Age (years) & & & & 0.169 \\
\hline$<50$ & 42 & 22 & 20 & \\
\hline$\geq 50$ & 86 & 34 & 52 & \\
\hline Tumor size $(\mathrm{cm})$ & & & & 0.052 \\
\hline$<4$ & 54 & 29 & 25 & \\
\hline$\geq 4$ & 74 & 27 & 47 & \\
\hline Location & & & & 0.681 \\
\hline Cardia & 50 & 23 & 27 & \\
\hline Body/antrum & 78 & 33 & 45 & \\
\hline Differentiation & & & & $<0.001^{\mathrm{a}}$ \\
\hline Well/moderate & 52 & 13 & 39 & \\
\hline Poor & 76 & 43 & 33 & \\
\hline Depth of invasion & & & & 0.907 \\
\hline $\mathrm{T} 1 / 2$ & 45 & 20 & 25 & \\
\hline $\mathrm{T} 3 / 4$ & 83 & 36 & 47 & \\
\hline Lymph node metastasis & & & & $0.029^{\mathrm{a}}$ \\
\hline Negative & 46 & 26 & 20 & \\
\hline Positive & 82 & 30 & 52 & \\
\hline TNM stage & & & & $<0.001^{\mathrm{a}}$ \\
\hline $\mathrm{I} / \mathrm{II}$ & 69 & 40 & 29 & \\
\hline III/IV & 59 & 16 & 43 & \\
\hline Distant metastasis & & & & 0.270 \\
\hline Negative & 120 & 54 & 66 & \\
\hline Positive & 8 & 2 & 6 & \\
\hline H. pylori infection & & & & 0.766 \\
\hline Negative & 36 & 15 & 21 & \\
\hline Positive & 92 & 41 & 51 & \\
\hline
\end{tabular}

FTO, fat mass and obesity associated; ${ }^{a} \mathrm{P}<0.05$ was considered statistically significant.

tumor size, location, distant metastasis and $H$. pylori infection $(\mathrm{P}>0.05)$.

To investigate the prognostic effect of FTO expression on overall survival rate of GC patients, Kaplan-Meier survival curves and the log-rank test was used to compare the 5-year survival rate of patients with high or low FTO expression level. According to the immunohistochemical results of FTO staining in tumors cells, GC patients were divided into two groups including FTO low expression group and high 

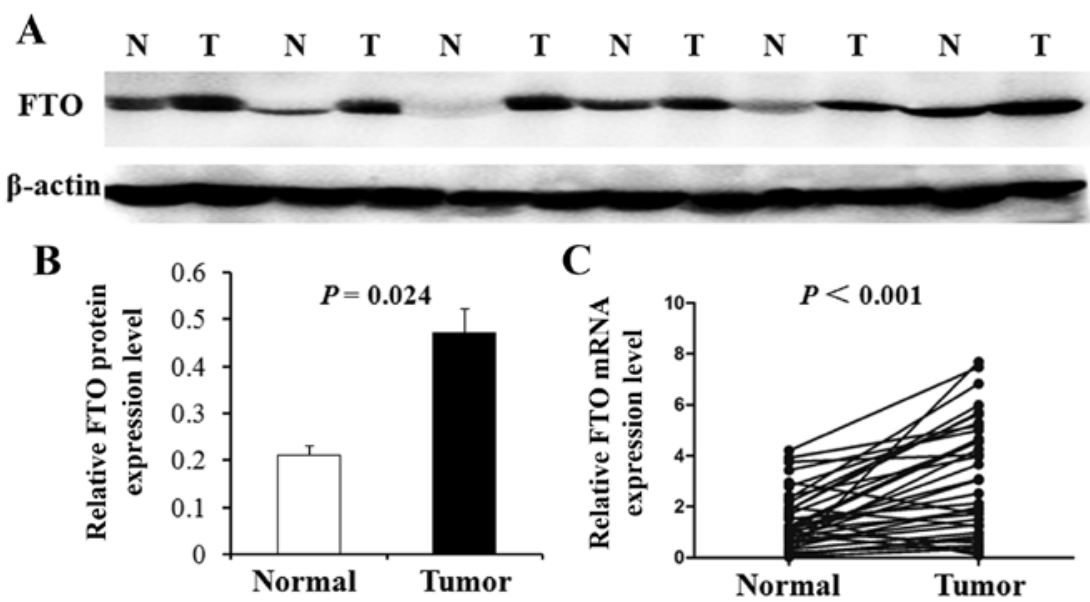

Figure 2. FTO gene expression is significantly upregulated in GC tissues. (A) Western blot analysis of six representative paired samples of GC (T) and their matched adjacent non-cancerous tissues $(\mathrm{N})$. Total proteins were prepared from tissue specimens obtained from gastric cancer and adjacent non-cancerous tissues, sequentially probed with anti-FTO and anti- $\beta$-actin antibodies. (B) Relative average FTO protein expression levels were remarkably upregulated in 18 of $24(75 \%)$ GC tissues compared with the corresponding adjacent non-cancerous tissues $(\mathrm{P}=0.024)$. (C) FTO mRNA expression level in GC tissues and paired adjacent non-cancerous tissues were determined by real-time RT-PCR and normalized to $\beta$-actin. The expression of FTO was significantly augmented in gastric carcinoma samples as compared with that in adjacent non-cancerous tissues $(\mathrm{P}<0.001)$.

Table III. Cox proportional hazards model analysis of prognostic factors.

\begin{tabular}{|c|c|c|c|c|c|c|}
\hline \multirow[b]{2}{*}{ Variables } & \multicolumn{3}{|c|}{ Univariate analysis } & \multicolumn{3}{|c|}{ Multivariate analysis } \\
\hline & HR & $95 \% \mathrm{CI}$ & P-value & HR & $95 \% \mathrm{CI}$ & P-value \\
\hline FTO expression (low vs. high) & 0.544 & $0.384-0.865$ & $<0.001^{\mathrm{a}}$ & 0.627 & $0.476-0.926$ & $<0.001^{\mathrm{a}}$ \\
\hline Sex (male vs. female) & 1.028 & $0.928-1.658$ & 0.580 & - & - & - \\
\hline Age (<50 vs. $\geq 50$ years) & 1.016 & $0.930-1.806$ & 0.925 & - & - & - \\
\hline Tumor size (<4 vs. $\geq 4 \mathrm{~cm}$ ) & 1.037 & $0.710-1.416$ & 0.064 & - & - & - \\
\hline Location (cardia vs. body/antrum) & 1.325 & $1.085-2.140$ & 0.904 & - & - & - \\
\hline Differentiation (poor vs. well/mod) & 0.863 & $0.526-1.376$ & $0.007^{\mathrm{a}}$ & 0.830 & $0.516-1.526$ & 0.353 \\
\hline Distant metastasis (+ vs. -) & 1.089 & $0.790-1.682$ & 0.626 & - & - & - \\
\hline Depth of invasion (T3/T4 vs. T1/T2) & 1.140 & $0.850-1.783$ & 0.072 & - & - & - \\
\hline TNM stage (III + IV vs. I + II) & 1.056 & $0.784-2.021$ & $0.002^{\mathrm{a}}$ & 0.949 & $0.650-1.458$ & $0.001^{\mathrm{a}}$ \\
\hline Lymph node metastasis (+ vs. -) & 1.956 & $1.264-2.560$ & $0.030^{\mathrm{a}}$ & 1.418 & $0.850-1.813$ & 0.552 \\
\hline
\end{tabular}

$\mathrm{HR}$, hazard ratio; $\mathrm{CI}$, confidence interval; ${ }^{\mathrm{a}} \mathrm{P}<0.05$ was considered statistically significant.

expression group. We found that the group with the high FTO expression levels had a poorer prognosis than the group with low levels of FTO expression $\left(\chi^{2}=8.415, \mathrm{P}<0.001\right.$; Fig. 3$)$.

Univariate and multivariate analysis was used to estimate the independent prognostic factors of FTO (Table III). The Cox's proportional hazards model showed that that histological grade, invasive depth, TNM stage, lymph node metastasis and FTO expression were significantly associated with overall survival in GC patients. In addition, we found that FTO expression and TNM stage were independent prognostic indicators for overall survival of GC patients.

Downregulation of FTO expression inhibits cell proliferation, migration and invasion abilities in vitro. Thus, based on the above, we hypothesized that FTO played the role as a cancer promoting gene. We used the MTT and colony formation assays to identify the effect of FTO on cell viability and proliferation. MKN45 cells have the highest FTO expression in the four GC cells (Fig. 4). After transfected with FTO siRNA1, FTO siRNA2 and FTO siNRA3, we found that siRNA1 reduced the level of endogenous FTO expression more significantly than siRNA2 and siRNA3 by western blot analysis (Fig. 5A). The results demonstrated that the viability of MKN45 cells was lower and the number of colonies of MKN45 cells in the siRNA treated group was less than in the control group (Fig. 5B and $\mathrm{C} ; \mathrm{P}<0.05)$. Besides, the Transwell assay shows that knockdown of FTO expression markedly decreased the migrated and invaded cell number of MKN45 cell lines (Fig. 5D and E; P<0.05).

Overexpression of FTO promotes cell viability, proliferation, migration and invasion in vitro. The effects of FTO on the 


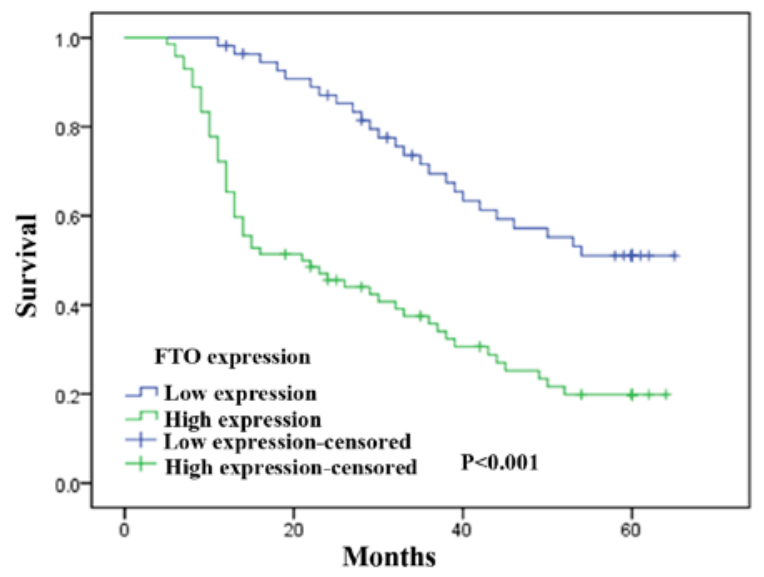

Figure 3. Association of FTO with patient prognosis in gastric cancer. Kaplan-Meier survival curves of the study population showing that patients with high FTO expression had a significantly poorer prognosis than those with low FTO expression $(\mathrm{P}<0.001$, log-rank test).
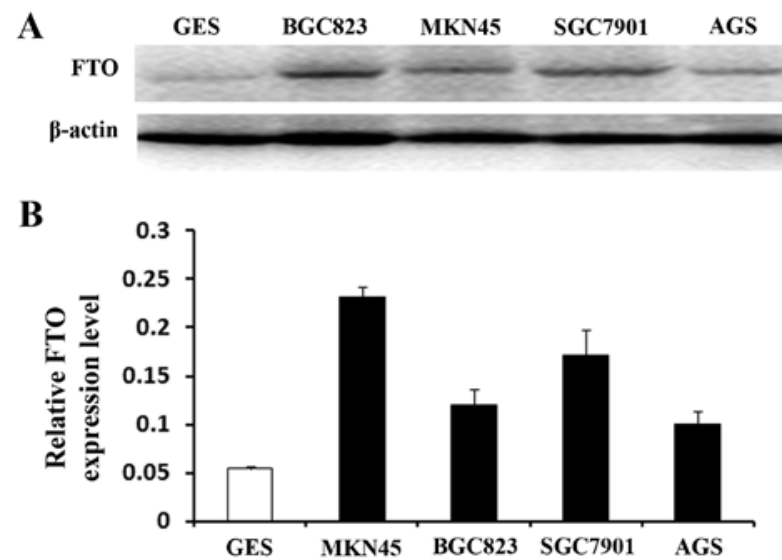

Figure 4(A,B). The FTO protein expression levels by western blot analysis in four GC cell lines and a gastric epithelial mucosa cell line (GES).

viability, proliferation, migration and invasion of GC cells were evaluated. AGS cell line was selected to investigate whether overexpression of FTO affected cell viability, proliferation, migration and invasion in GC. In addition, the FTO expression level of AGS cell line was the lowest in the four GC cell lines. Thus, AGC cells were transfected with recombinant plasmids containing the full ORF of the wild-type FTO. Western blot analysis confirmed that the cells transfected with the FTO recombinant plasmid showed higher expression levels of the FTO protein compared with cells transfected with the empty vector control pEGFP-N1 (Fig. 6A). The results of MTT and colony formation assays showed that overexpression of FTO promoted viability and the number of colonies of AGS cells (Fig. 6B and C; $\mathrm{P}<0.05$ ). We also found that overexpression of FTO could increase the transformation phenotype of GC cells in vitro. Then, to investigate the potential affect of FTO on cellular migration and invasion, FTO overexpressing plasmid or control plasmid were transfected into AGS cells. We use Transwell assays to determine their migratory and invasive abilities after $24 \mathrm{~h}$. The result reflects that overexpression of FTO enhanced the migratory and invasive capacity of AGS cells (Fig. 6D and E; $\mathrm{P}<0.05$ ).
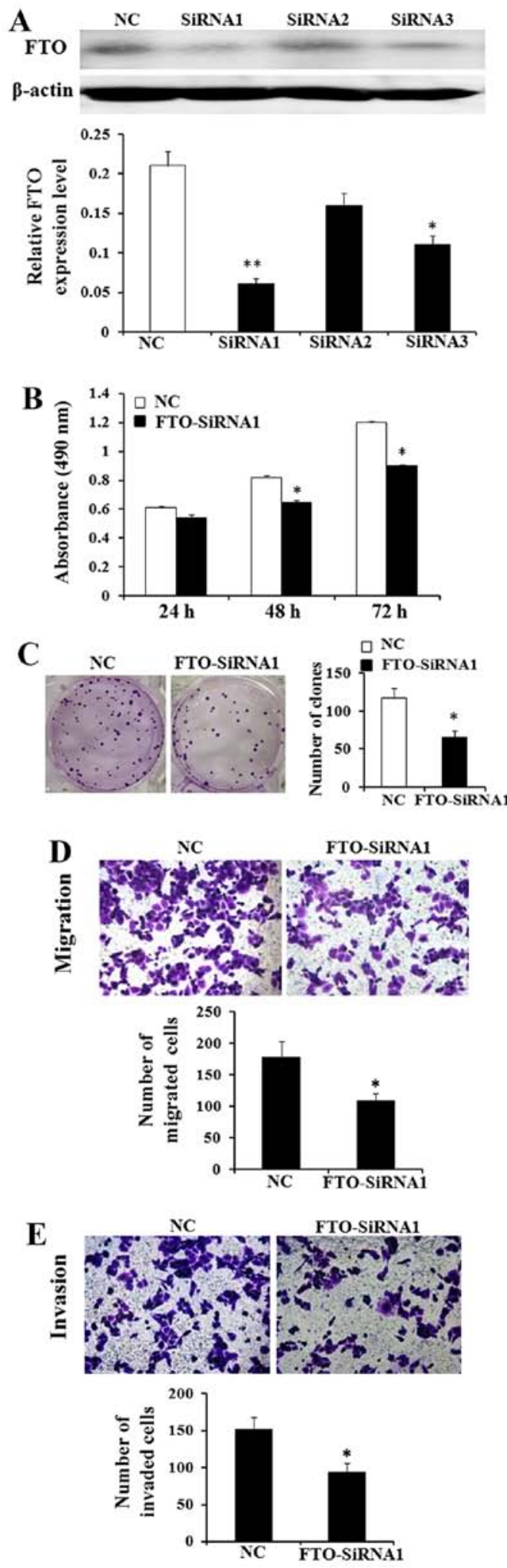

Figure 5. Knockdown of FTO expression inhibits GC cell viability, proliferation, migration and invasion in vitro by using siRNA. (A) Western blot analysis of FTO protein expression levels. The three different FTO siRNA transfectants (siRNA1, siRNA2 and siRNA3) and the empty vector transfectant (NC) are shown. (B) Cell viability was evaluated with MTT assay using absorbance readings at $490 \mathrm{~nm}$. MKN45 cells were transfected with FTO siRNA or empty vector for 1,2 and 3 days, respectively. The values are indicated by the mean of three determinations. (C) Colony numbers of the MKN45/siRNA and control cells in plate colony formation assays. The data represent the mean \pm SEM of three independent experiments. (D and E) Transwell assays demonstrated that silencing of FTO expression by siRNA inhibited cell migration and invasion in MKN45 cells. Representative images of stained cells are shown with the original magnification of $\mathrm{x} 100$. ${ }^{*} \mathrm{P}<0.05$. 
A

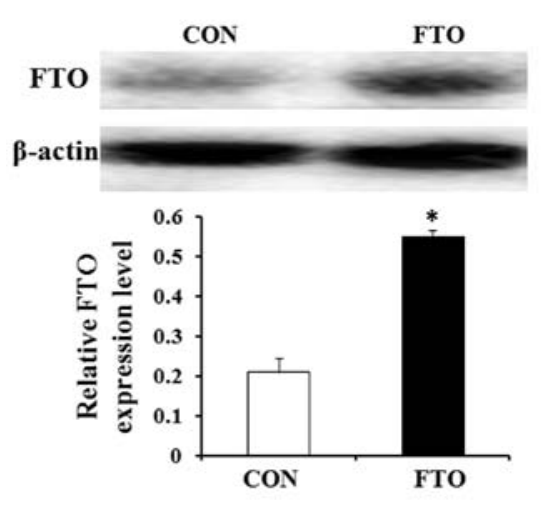

$\mathbf{B}$

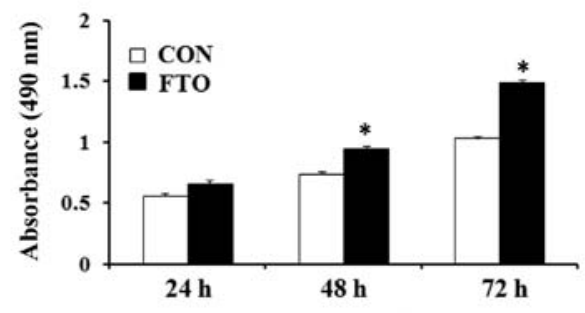

C

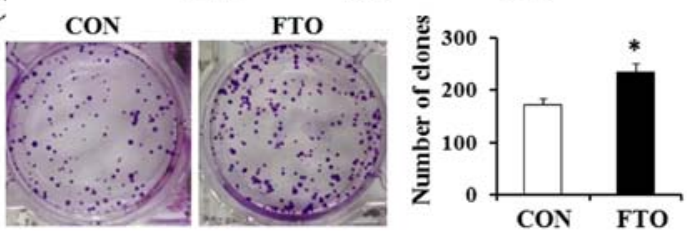

$\mathbf{E}$

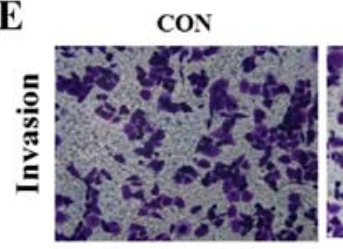

FTO
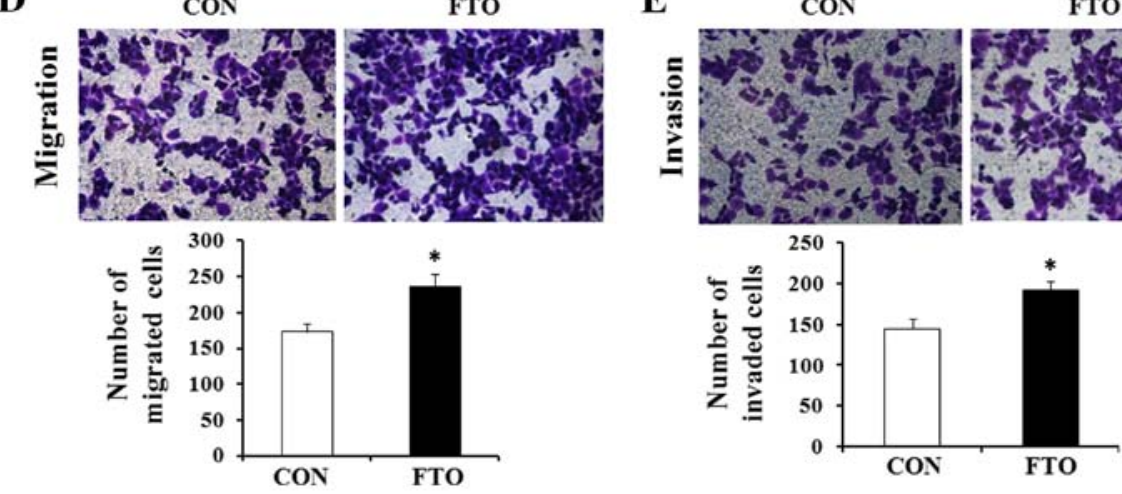

Figure 6. Overexpression of FTO promotes the viability, proliferation, migration and invasion of GC cells in vitro. (A) Western blot analysis of FTO protein expression levels. The FTO overexpression transfectant (FTO) and the empty vector transfectant (CON) are shown. (B) Cell viability was evaluated with MTT assay using absorbance readings at $490 \mathrm{~nm}$. AGS cells were transfected with pcDNA 3.1(+)-FTO or empty vector for 1, 2 and 3 days, respectively. The values are indicated by the mean of three determinations. (C) Colony numbers of the AGS/FTO and control cells in plate colony formation assays. The data represent the means \pm SEM of three independent experiments. (D and E) Transwell assays demonstrated that overexpression of FTO in AGS cells transfected with pcDNA3.1(+)-FTO plasmid promoted cell migration and invasion compared with empty vector. Representative photos of stained cells are shown with the original magnification of $\mathrm{x} 100 .{ }^{*} \mathrm{P}<0.05$.

\section{Discussion}

Despite the incidence of gastric cancer is declining, gastric cancer imposes a significant health burden around the world. GC is often diagnosed in advanced stages and carries a poor prognosis (17). At present, gastric resection combined with chemotherapy and/or radiotherapy is the rational treatment. But early diagnosis and treatment are keys for better clinical outcome in with GC patients (18). Owing to lack of effective biomarkers for early gastric cancer, the outcome of gastric cancer patients remains dismal (19). Discovery of new biomarkers can help us find early and accurate prediction of tumor behavior, and the survival of GC patients can be improved. By taking advantage of serum protein antigens, oncogenic genes or gene families through improving molecular biological technologies, modern biomedical research has explored many potential gastric cancer biomarker genes $(19,20)$. FTO is a new gene as a biological marker.

FTO is mapped on chromosome $16 \mathrm{p} 12.2$ and is widely expressed in many tissues of human body, with highest levels are detected in the brain, pancreatic islets, and the digestive organs (12). It is proved that FTO gene is link to obesity, dysmorphic facies and other diseases (21). The function of the FTO gene product and the biological pathways involved are not completely known. Furthermore, it has been shown that
FTO localizes into the nucleus and functional studies with bioinformatics analysis revealed that the FTO gene encodes a 2-oxoglutarate-dependent nucleic acid demethylase which may have a potential role in regulating the transcription of genes related to in metabolism by nucleic acid demethylation of DNA (22). FTO gene is a nuclear protein of the AlkB related non-heme iron, however, the precise physiological function of the gene is unclear. What we do know is that other non-heme iron enzymes function to reverse alkylated DNA and RNA damage by oxidative demethylation (23). It has been suggested that increased expression of FTO can promote the occurrence and development of various types of cancer. Such as breast, thyroid, endometrial cancer (14-16), and experiments suggest FTO expression positively correlates with these cancers. Thus, we can speculate that FTO acts as a tumor promoting gene. There are no studies showing the expression profile of FTO in gastric cancer. The above experiments were designed to predicate the role of FTO in GC.

In the present study, the FTO protein expression was detected by western blot analysis. We found that FTO protein was upregulated in the GC tissues compared with the carcinoma adjacent tissues. Moreover, we use quantitative PCR to analyze the mRNA level of FTO. PCR results showed that the mRNA levels of FTO in the GC tissues were obvi- 
ously higher compared with the corresponding carcinoma adjacent tissues. To further confirm the results of western blot analysis and qPCR, we used immunohistochemical staining to examine the FTO expression in GC tissues and to estimate whether the FTO expression level has links with clinicopathological parameters and the prognosis of GC patients. We also found that FTO expression in GC tissues was significantly higher than the adjacent non-cancerous tissues. In addition, FTO expression was positively correlated with histological differentiation, lymph node metastasis and TNM stage in GC. Therefore, as well as in breast, thyroid and endometrial cancer, FTO overexpression may promote the occurrence of GC and the abnormal expression of FTO might be associated with GC tumor progression and metastasis. Moreover, in vitro, the viability, proliferation and migration and invasion of AGS cells were markedly promoted by overexpression of FTO, however, knockdown of FTO expression inhibited the viability, proliferation and migration and invasion of MKN45 cells. It is generally known that $H$. Pylori infection has been considered as a risk factor for the occurrence of GC, $50 \%$ of the world's population is infected with $H$. Pylori (24). Nevertheless, in the present study, no significant difference in FTO expression was observed in patients with or without $H$. pylori infection.

According to the Kaplan-Meier analysis, the overall 5-year survival of GC patients and the level of FTO expression were negatively correlated. High FTO expression had a significant relationship with shorter survival time of GC patients. Based on the above experimental results, we can conjecture that FTO may play more vital role in advanced cancer of the stomach and the FTO protein may have a direct effect on the invasion of GC. Multivariate analysis indicated that in overall survival of GC patients, FTO expression was an independent prognostic indicator, and high expression of FTO gene may indicate poor prognosis. Recently, FTO has been shown to contribute to the development of several cancer types (14-16). Although in these reported studies, the exact functions and the mechanistic actions of FTO have not been defined completely so far.

In conclusion, our data indicate that the overexpression of FTO is involved in cancer progression and dedifferentiation in gastric carcinoma patients and the detection of increased FTO expression might help confirm GC patients with a poor prognosis. However, the exact mechanism of FTO gene in gastric cancer and other tumors is not completely understood. Therefore, further large studies are needed to figure out the relationship of FTO expression with GC and to investigate the mechanisms underlying the relationship.

\section{Acknowledgements}

The present study was funded by a grant from the Nantong Science and Technology Project (MS22016033).

\section{References}

1. Mcguire S: World Cancer Report 2014. Geneva, Switzerland: World Health Organization, International Agency for Research on Cancer, WHO Press, 2015. Adv Nutr 7: 418-419, 2016.

2. Torre LA, Bray F, Siegel RL, Ferlay J, Lortet-Tieulent J and Jemal A: Global cancer statistics, 2012. CA Cancer J Clin 65: 87-108, 2015.
3. Jackson CB and Giraud AS: STAT3 as a prognostic marker in human gastric cancer. J Gastroenterol Hepatol 24: 505-507, 2009.

4. Tahara T, Shibata T, Nakamura M, Yamashita H, Yoshioka D, Okubo M, Yonemura J, Maeda Y, Maruyama N, Kamano T, et al: Association between IL-17A, -17F and MIF polymorphisms predispose to $\mathrm{CpG}$ island hyper-methylation in gastric cancer. Int J Mol Med 25: 36-54, 2010.

5. Compare D, Rocco A and Nardone G: Risk factors in gastric cancer. Eur Rev Med Pharmacol Sci 14: 302-308, 2010.

6. González CA and Agudo A: Carcinogenesis, prevention and early detection of gastric cancer: Where we are and where we should go. Int J Cancer 130: 745-753, 2012.

7. Resende C, Ristimäki A and Machado JC: Genetic and epigenetic alteration in gastric carcinogenesis. Helicobacter 15 (Suppl 1): 34-39, 2010.

8. Kurowski MA, Bhagwat AS, Papaj G and Bujnicki JM: Phylogenomic identification of five new human homologs of the DNA repair enzyme AlkB. BMC Genomics 4: 48, 2003.

9. Peters T, Ausmeier K, Dildrop R and Rüther U: The mouse Fused toes $(\mathrm{Ft})$ mutation is the result of a $1.6-\mathrm{Mb}$ deletion including the entire Iroquois B gene cluster. Mamm Genome 13: 186-188, 2002.

10. Gerken T, Girard CA, Tung YC, Webby CJ, Saudek V, Hewitson KS, Yeo GSH, McDonough MA, Cunliffe S, McNeill LA, et al: The obesity-associated FTO gene encodes a 2-oxoglutarate-dependent nucleic acid demethylase. Science 318: 1469-1472, 2007.

11. Jia G, Yang CG, Yang S, Jian X, Yi C, Zhou Z and He C: Oxidative demethylation of 3-methylthymine and 3-methyluracil in single-stranded DNA and RNA by mouse and human FTO. FEBS Lett 582: 3313-3319, 2008

12. Frayling TM, Timpson NJ, Weedon MN, Zeggini E, Freathy RM, Lindgren CM, Perry JR, Elliott KS, Lango H, Rayner NW, et al: A common variant in the FTO gene is associated with body mass index and predisposes to childhood and adult obesity. Science 316: 889-894, 2007.

13. Hernández-Caballero ME and Sierra-Ramírez JA: Single nucleotide polymorphisms of the FTO gene and cancer risk: An overview. Mol Biol Rep 42: 1-6, 2014.

14. Tan A, Dang Y, Chen G and Mo Z: Overexpression of the fat mass and obesity associated gene (FTO) in breast cancer and its clinical implications. Int J Clin Exp Pathol 8: 13405-13410, 2015.

15. Sigurdson AJ, Brenner AV, Roach JA, Goudeva L, Müller JA, Nerlich K, Reiners C, Schwab R, Pfeiffer L, Waldenberger M, et al: Selected single-nucleotide polymorphisms in FOXE1, SERPINA5, FTO, EVPL, TICAM1 and SCARB1 are associated with papillary and follicular thyroid cancer risk: Replication study in a German population. Carcinogenesis 37: 677-684, 2016.

16. Zhu Y, Shen J, Gao L and Feng Y: Estrogen promotes fat mass and obesity-associated protein nuclear localization and enhances endometrial cancer cell proliferation via the mTOR signaling pathway. Oncol Rep 42: 381-385, 2016.

17. Wadhwa R, Song S, Lee JS, Yao Y, Wei Q and Ajani JA: Gastric cancer-molecular and clinical dimensions. Nat Rev Clin Oncol 10: 643-655, 2013.

18. Piazuelo MB and Correa P: Gastric cancer: Overview. Colomb Med 44: 192-201, 2013.

19. Wu HH, Lin WC and Tsai KW: Advances in molecular biomarkers for gastric cancer: miRNAs as emerging novel cancer markers. Expert Rev Mol Med 16: 53-62, 2014.

20. Fan B, Zhang LH, Jia YN, Zhong XY, Liu YQ, Cheng XJ, Wang XH, Xing XF, Hu Y, Li YA, et al: Presence of S100A9positive inflammatory cells in cancer tissues correlates with an early stage cancer and a better prognosis in patients with gastric cancer. BMC Cancer 12: 316, 2011

21. Tschritter O, Preissl H, Yokoyama Y, Machicao F, Häring HU and Fritsche A: Variation in the FTO gene locus is associated with cerebrocortical insulin resistance in humans. Diabetologia 50: 2602-2603, 2007.

22. Melnik BC: Milk: An epigenetic amplifier of FTO-mediated transcription? Implications for Western diseases. J Transl Med 13: $1-22,2015$.

23. Raskandersen M, Almén MS and Schiöth HB: Scrutinizing the FTO locus: Compelling evidence for a complex, long-range regulatory context. Hum Genet 134: 1-11, 2016.

24. Brown LM: Helicobacter pylori: Epidemiology and routes of transmission. Epidemiol Rev 22: 283-297, 2000. 